\title{
Physiological Mechanisms of Onset Adaptation and Contralateral Suppression of DPOAEs in the Rat
}

\author{
E. M. Relkin, ${ }^{1,2}$ A. Sterns, ${ }^{1}$ W. Azeredo, ${ }^{3}$ B. A. Prieve, ${ }^{1,4}$ and C. I. Woods ${ }^{1,3}$ \\ ${ }^{1}$ Institute for Sensory Research, Syracuse University, Syracuse, NY, USA \\ ${ }^{2}$ Bioengineering and Neuroscience, Syracuse University, Syracuse, NY, USA \\ ${ }^{3}$ State University of New York Upstate Medical Center, Syracuse, NY, USA \\ ${ }^{4}$ Department of Communication Sciences and Disorders, Syracuse University, NY, USA
}

Received: 15 August 2004; Accepted: 15 December 2004; Online publication: 10 June 2005

\begin{abstract}
An investigation was undertaken to measure medial olivocochlear (MOC) reflexes in anesthetized rats before and after sectioning of the middle-ear muscles. Distortion product otoacoustic emission (DPOAE) magnitude and phase temporal responses were measured ipsilaterally to study MOC-mediated "DPOAE onset adaptation" and in the presence of a contralateral noise to study MOC-mediated contralateral "suppression" (terms as used by previous researchers). Distortion product otoacoustic emission onset adaptation and contralateral suppression had predictable changes in direction of magnitude and phase that were dependent on the input-output function. After sectioning of the middle-ear muscles (MEMs), DPOAE onset adaptation and contralateral suppression were greatly reduced, and there were little, if any, changes in phase. These "residual" changes were interpreted as a result of the MOC reflex. The results suggest that what appears to be DPOAE onset adaptation and contralateral suppression can be mediated primarily by MEM reflexes. When studying MOC effects on otoacoustic emissions (OAEs) using acoustic stimulation, it is necessary to make recordings over a span of stimulus levels. In
\end{abstract}

\footnotetext{
This manuscript was based on a rough draft written by the first author and many discussions with him prior to his death. Every effort has been made to portray the data and its discussion as he would have intended. BAP, ARS, CIW.

Correspondence to: Beth A. Prieve - Department of Communication Sciences and Disorders • 805 S. Crouse Avenue Syracuse, NY 132442280, USA. Telephone: (315) 443-9614; fax: (315) 443-1113; email: baprieve@syr.du
}

addition, looking at both magnitude and phase of the OAE may help separate what is due to the MOC reflex from MEM reflex.

Keywords: DPOAEs, medial olivocochlear reflexes, middle-ear muscle reflexes, rats

\section{INTRODUCTION}

Cell bodies of the medial olivocochlear (MOC) efferent system are located near the medial superior olive and project in two pathways. A proportion of the fibers project to hair cells on the ipsilateral side (which shall be referred to as the "uncrossed" fibers), and a proportion project to hair cells on the contralateral side (referred to as the "crossed" fibers). The influence of the MOC system on auditory processing has been researched in many ways, one of them by observing changes in otoacoustic emissions (OAEs). The first studies of reduction in otoacoustic emissions (OAEs) during stimulation of the contralateral ear (investigating the uncrossed MOC) were published in the early 1990s (e.g., Collet et al. 1990; Veuillet et al. 1991), and the term "contralateral suppression" has been used to identify this phenomenon.

One technique that has been used to study the crossed MOC track is to measure the temporal waveform of the DPOAE. Liberman et al. (1996) first described changes in the amplitude of DPOAEs in the cat at stimulus onset that were largely monotonic with time, had a time constant of approximately $100 \mathrm{~ms}$, and changed by as much as $9 \mathrm{~dB}$ from the 
beginning to the end of the onset response. The authors referred to this phenomenon as "fast onset adaptation," and it was subsequently reported in mice (Sun and Kim 1999; McGee et al. 2000; Jacobson et al. 2003), gerbils (McGee et al. 2000), guinea pigs (Maison and Liberman 2000; Kujawa and Liberman 2001; Lukashkin and Russell 2002), and humans (Kim et al. 2001). Whereas adaptation is most usually thought of as decreasing as a function of time, onset adaptation of the DPOAE can also be observed as increasing with time (Maison and Liberman 2000; Kujawa and Liberman 2001; Lukashkin and Russell 2002).

Liberman et al. (1996) showed that the direction of DPOAE onset adaptation for a given set of primary stimuli was the same as the direction of the DPOAE change when a noise was presented to the contralateral ear (whether the noise produced an increase or a decrease in the DPOAE) suggesting a common underlying mechanism. Controlled studies in cats and guinea pigs suggest that contralateral suppression and DPOAE onset adaptation in these species are a result of the MOC reflex and not the MEMR (Puel and Rebillard 1990; Kujawa and Liberman 2001; Liberman et al. 1996). In contrast, contralateral suppression and onset adaptation in rabbits are mainly attributed to MEMR effects. Whitehead et al. (1991) indirectly tested DPOAE reduction because of contralateral acoustic stimulation in awake rabbits. In addition, they measured MEMR thresholds using a clinical device. They found that the range of frequencies and levels producing MEMR activation were the same as those for which changes in DPOAEs were seen and concluded that MEMR activation was the cause for the reduction in DPOAEs. Luebke et al. (2002), using changes in the sound pressure level of the primary stimuli to deduce that the MEMR was activated, found that onset adaptation in awake rabbits was primarily mediated by the MEMs.

Because it is uncertain in some species or experimental protocols whether observed effects are a result of the MOC reflex, MEMR, or a possible combination of both, it seems appropriate to define the observed effects without reference to their underlying physiological mechanism. In this paper, the abbreviation " $\Delta \mathrm{LI}$ " stands for changes in level of the ipsilaterally recorded DPOAE over the first few hundred milliseconds of the response, the same time frame over which DPOAE onset adaptation is observed. The abbreviation " $\Delta \mathrm{PI}$ " refers to phase changes during the same time frame. The abbreviation " $\Delta \mathrm{LC}$ " stands for changes in DPOAEs during acoustic stimulation of the contralateral ear, and " $\triangle \mathrm{PC}$ " stands for changes in phase during the same time frame. The previously used terms "DPOAE onset adaptation" and "contralateral suppression" will be reserved for those situations where there is reasonable evidence that the phenomena are a result of the MOC reflex. The goal of the present study was to compare $\Delta \mathrm{LI}$ and $\Delta \mathrm{LC}$ in rats, before and after cutting of middle-ear muscles, to characterize the underlying physiological mechanisms responsible for the changes.

\section{METHODS}

\section{Subjects}

Subjects were nine Sprague-Dawley male rats obtained from a licensed breeder. Rats were chosen as the experimental animal because a stereotaxic atlas of the brain is available for this species, and one component of our research is to compare changes of OAEs with electric and acoustic stimulation of the MOC reflex. The rats were retired breeders weighing $450-650 \mathrm{~g}$. The rats were anesthetized with a mixture of acepromazine $(0.48 \mathrm{mg} / \mathrm{ml})$ and ketamine $(95$ $\mathrm{mg} / \mathrm{ml}$ ) administered i.m. at a starting dose of $2 \mathrm{ml} /$ $\mathrm{kG}$. Atropine $(0.4 \mathrm{mg} / \mathrm{kG})$ was also administered at the start of the experiment to control for respiratory secretions. Supplemental doses of anesthesia of one fifth of the original bolus were administered approximately once per hour or when the rat responded to a toe pinch. Throughout surgery and data collection, rectal body temperature was maintained at $38^{\circ} \mathrm{C}$ by a feedback-controlled heating pad.

The animal facilities have full accreditation from Association for Assessment and Accreditation of Laboratory Animal Care. All experimental procedures were approved by the local Institutional Animal Care and Use Committee and met the National Institutes of Health guidelines for animal care.

\section{Equipment}

Figure 1 shows a schematic diagram of the equipment. A custom system was designed to measure the temporal envelope of the DPOAEs. The system was similar to that described by Rosowski et al. (1984), in which a lock-in amplifier was used to measure the inphase and quadrature components of the DPOAE as a function of time. The temporal envelopes of the magnitude and phase of the DPOAEs were recorded. The temporal resolution of the recording was limited by the time-constant setting of the lock-in amplifier which was set to $3 \mathrm{~ms}$.

Primaries ( $\mathrm{fl}$ and $\mathrm{f2}$ ) were sinusoidal waveforms generated by two, identical, digital oscillators. One oscillator generated the lower-frequency primary (f1), and a second oscillator generated the higherfrequency primary (f2). The ratio of the primaries 


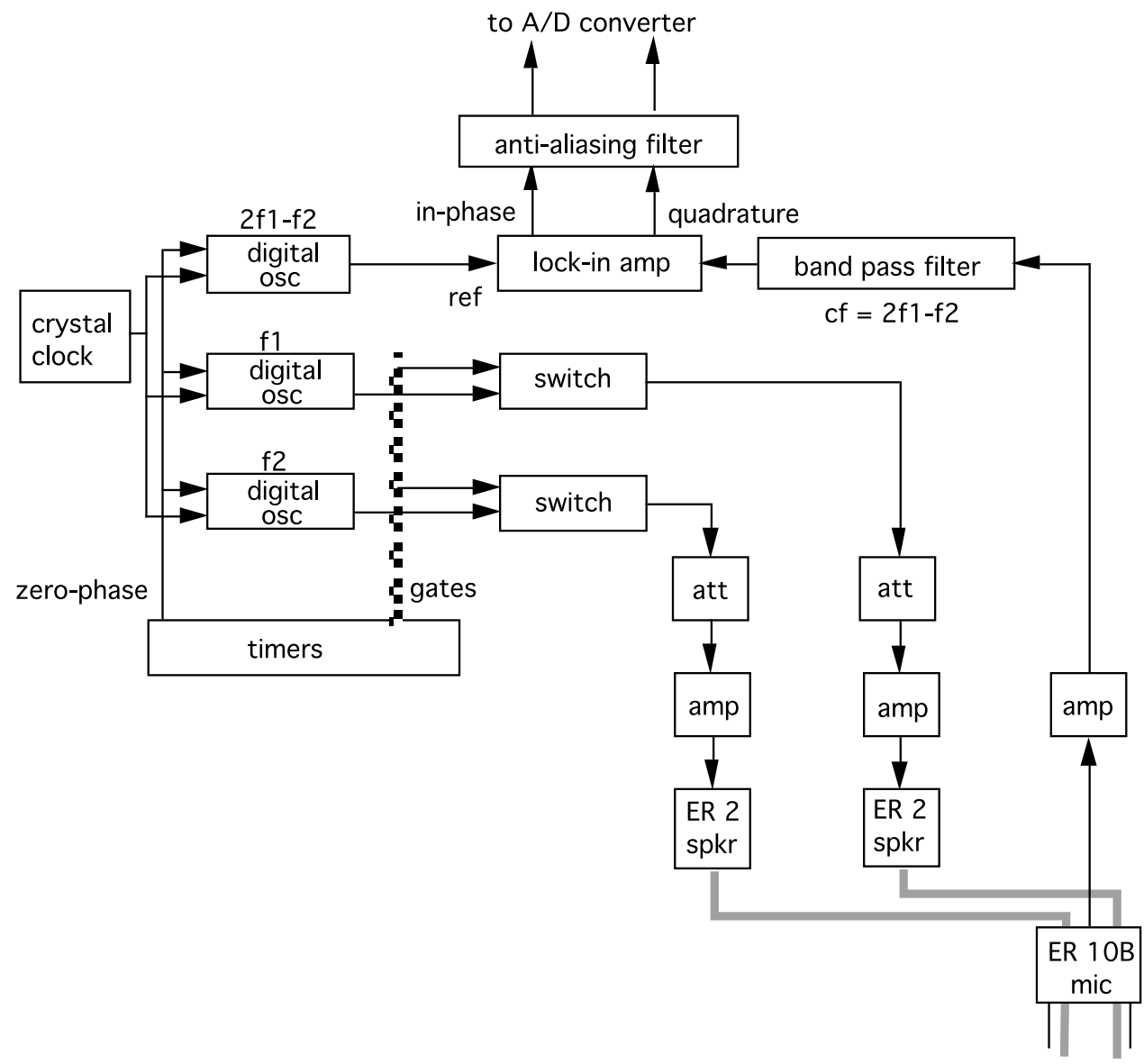

FIG. 1. Schematic diagram of stimulus and recording equipment. (f2/f1) was 1.25. A third, identical digital oscillator was used to tune the lock-in amplifier to the DPOAE frequency. All three oscillators were driven by a common signal derived from an $8-\mathrm{MHz}$ crystal-controlled clock, and the oscillators were reset simultaneously to zero phase at the beginning of each stimulus presentation, thus insuring near-perfect synchrony with zero phase drift. The frequencies of the oscillators could be set with millihertz resolution.

The primary stimuli were $500 \mathrm{~ms}$ in duration, and the onsets and offsets were shaped by cosine-squared functions with a rise/fall of $5 \mathrm{~ms}$. The rise/fall was chosen as the lowest value that allowed adequate rejection of any "artifacts" associated with the onset and offset of gated primary stimuli for the range of DPOAE frequencies and the $\mathrm{f} 1 / \mathrm{f} 2$ ratio used in this study. The primary stimuli were then attenuated, amplified, and delivered to the subjects through ER-2 earphones.

To study $\triangle \mathrm{LC}$, a broadband noise was presented to the opposite ear also using an Etymotic ER-2 speaker. The noise level was calibrated in a $1-\mathrm{cm}^{3}$ coupler using a Bruel and Kjaer, 0.5-in. microphone but was not otherwise calibrated for each individual animal. The noise was $200 \mathrm{~ms}$ in duration and began $50 \mathrm{~ms}$ after the onset of the f2 primary stimulus. The level of the broadband noise was $60 \mathrm{~dB}$ SPL, except in one animal, in which the noise was $70 \mathrm{~dB}$ SPL. Early in the experimentation, we tried several levels of noise and ultimately settled on $60 \mathrm{~dB}$ SPL because we saw adequate changes, but we were hoping to minimize MEM involvement using the lower noise level.

To measure DPOAEs, sound pressure in the ear canal was recorded with the microphone of an Etymotic ER-10B+ probe. The sound in the ear canal was amplified by $40 \mathrm{~dB}$ and then filtered. To measure the time course of the DPOAE, it is necessary to separate the sound pressure produced by the DPOAE from the sound pressure produced by the primary stimuli. This signal processing can be difficult because of the relative closeness of the frequency of the DPOAE to the primary frequencies and because the intensity of the DPOAE is typically $40 \mathrm{~dB}$ or lower than the intensities of the primary stimuli. The following example, using conditions encountered in our study, illustrates the quality of filtering that is required to isolate the DPOAE. For a DPOAE frequency of $3.6 \mathrm{kHz}$, using primary stimuli of frequency 4.8 and $6.0 \mathrm{kHz}$ (frequency ratio of 1.25 ), if the DPOAE is $20 \mathrm{~dB}$ SPL when the lower frequency primary is at $80 \mathrm{~dB}$ SPL, attenuating that primary to a level $20 \mathrm{~dB}$ below that of the DPOAE requires a 
rejection slope of $-240 \mathrm{~dB} /$ octave. The problem of separating the signals in the frequency domain is compounded by any frequency splatter that occurs at onset and offset when the primary stimuli are gated. The $\mathrm{f} 2 / \mathrm{fl}$ ratio of 1.25 and rise/fall time of the cosine-squared gating allowed for nearly complete separation of the DPOAE from the nearest primary stimulus, f1, including any frequency splatter associated with the onset of $\mathrm{fl}$. Because $\mathrm{f2}$ was further away from the DPOAE frequency, it was of less concern in this regard.

The technique for separating the DPOAE from the primaries consisted of a combination of analog and lock-in filtering followed by signal averaging. After amplification of the recording microphone, the output was band-pass filtered at the DPOAE frequency that included one stage of high-pass filtering and three stages of low-pass filtering. The high-pass rejection slope was $48 \mathrm{~dB}$ /octave, and the total lowpass rejection slope was $228 \mathrm{~dB}$ /octave (measured at twice the center frequency). The filtered output was sent to a lock-in amplifier. In-phase and quadrature components were averaged independently with the time constant on the lock-in amplifier set to $3 \mathrm{~ms}$. Stimuli were repeated every $2 \mathrm{~s}$, and the average
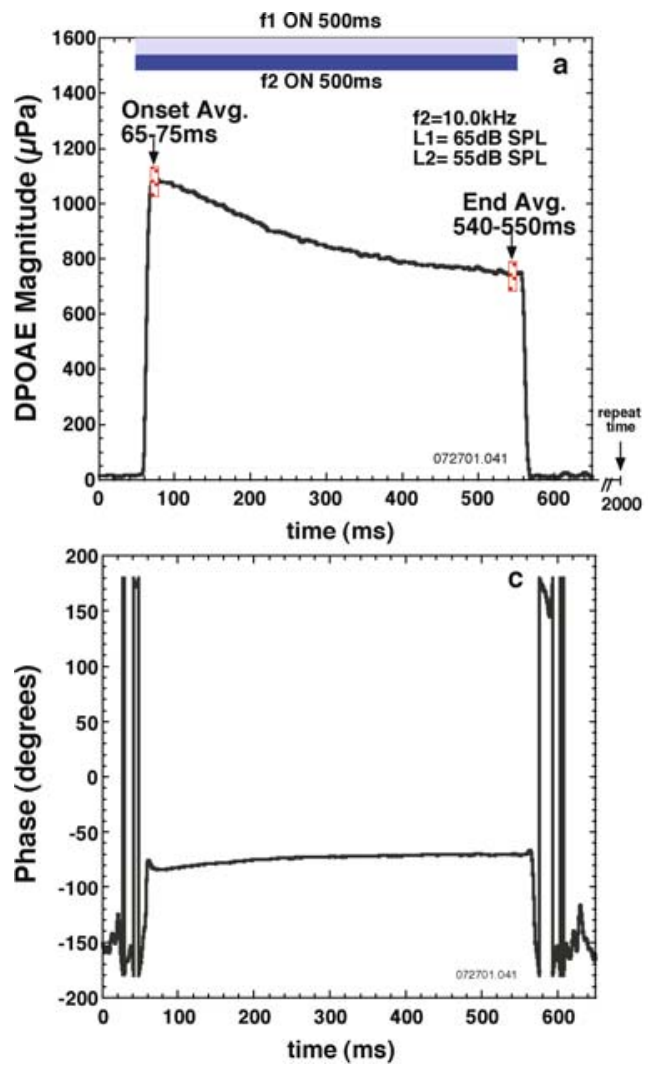

FIG. 2. Example of DPOAE magnitude (panel a) and phase (panel c) temporal responses using the switched $\mathrm{f} 1$ condition, which was used to measure $\Delta \mathrm{LI}$. Panels $\mathbf{b}$ and $\mathbf{d}$ illustrate DPOAE temporal responses using the continuous $\mathrm{f} 1$ condition, which was used to consisted of 50 stimulus repetitions. The in-phase and quadrature outputs of the lock-in amplifier were low-pass filtered to provide anti-aliasing and then were sampled by a computer-controlled A/D converter at 10,000 samples/s. At the end of each stimulus condition, magnitude, i.e., the temporal envelope, and phase were calculated from the averaged components and stored for off-line analysis.

\section{Stimulus timing}

Two stimulus conditions were used to observe the temporal properties of the DPOAE. To record $\Delta \mathrm{LI}, \mathrm{f} 1$ was switched simultaneously with f2 for what we refer to as the switched $f 1$ condition. The continuous $f 1$ condition was used to study $\triangle \mathrm{LC}$ and, except for control conditions, included the presentation of a contralateral broadband noise. Figure 2 summarizes the timing of the stimuli for these two conditions and shows example magnitude and phase recordings for $\Delta \mathrm{LI}$ (panels a and c) and $\Delta \mathrm{LC}$ (panels b and d).

As indicated in the schematic in Figure 2a, fl and f2 were switched on simultaneously during the switched fl condition. All onset and offset phenomena could be observed, including those associated
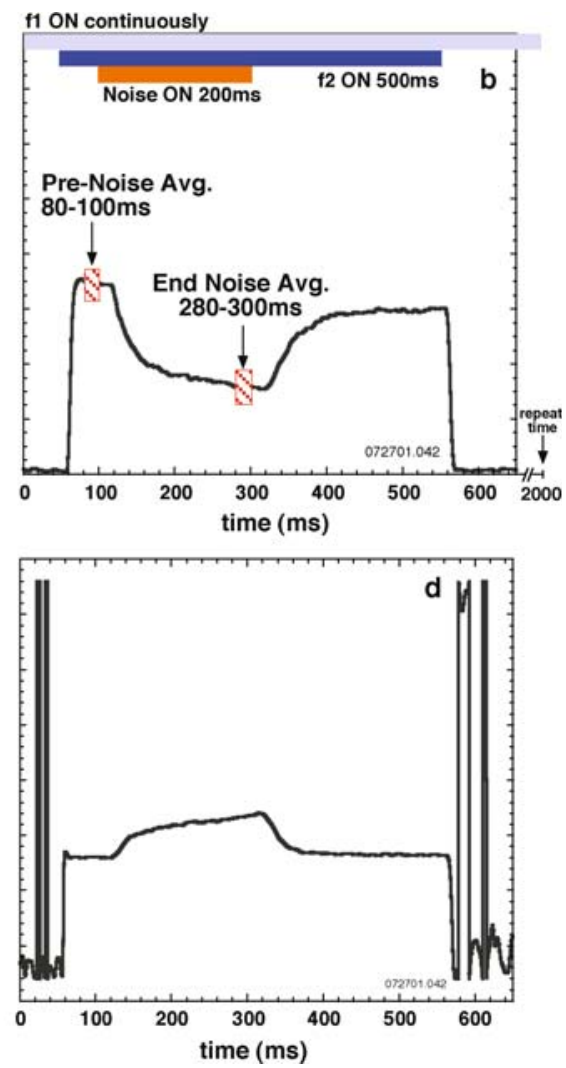

measure $\Delta \mathrm{LC}$. The gray bars at the top of the panels illustrate timing of the primaries. The hatched areas on the magnitude temporal responses (panels $\mathbf{a}$ and $\mathbf{b}$ ) illustrate the time periods over which measurements were taken to quantify $\Delta \mathrm{LI}$ and $\Delta \mathrm{LC}$. 
with ipsilateral activation of either the MOC or MEM reflex. Figure $2 \mathrm{a}$ shows the DPOAE magnitude as a function of time along with a schematic of the timing of the stimulus presentations. In panel $c$, the corresponding phase is shown. Using a 2-s interstimulus interval, each averaged response was largely independent of the previous response. Preliminary data showed that as much as $10 \mathrm{~s}$ was needed to achieve absolute independence, but $2 \mathrm{~s}$ was found to be an adequate compromise that provided more practical data collection times.

The continuous fl condition (Figure 2b) was used to observe DPOAE changes to the gated, contralateral broadband noise. Because the continuous f1 primary was typically $10 \mathrm{~dB}$ greater than $\mathrm{f} 2$, timedependent phenomena associated with ipsilateral responses to the primaries, such as activation of the MEM or MOC reflex, should be in a "steady-state" condition when the f2 stimulus is turned on to evoke the DPOAE. Thus the temporal envelope of the DPOAE was observed to be similar to the envelope of the gated $\mathrm{f} 2$ primary, with the possible exception of very rapid onset and offset transients (duration of approximately $10 \mathrm{~ms}$ ) similar to those described by Talmadge et al. (1999). Those authors attributed the transients to the interactions of multiple DPOAE sources with differing magnitudes, phases, and latencies. These phenomena were not of primary interest in this study. Presentation of the contralateral noise during the "flat" portion of the temporal envelope of the DPOAE made it easier to observe and quantify the time course of the response to the noise. Panel $d$ shows the corresponding phase plotted as a function of time.

Overlaid on the magnitude functions (Figure 2a and b) are hatched areas that indicate the temporal windows that were used to quantify respective changes in the magnitude and phase of the DPOAE. The $\Delta \mathrm{LI}$ (panel a) was quantified as the difference between the average of the DPOAE magnitude, in $\mathrm{dB}$ SPL, in a 10-ms window 65-75 ms after stimulus onset and the average of the magnitude, also in $\mathrm{dB}$ SPL, for a window of equal duration from 540 to $550 \mathrm{~ms}$ after stimulus onset. Similarly, $\Delta$ LC (panel b) was quantified as the difference between the average magnitude measured in a 20-ms window near stimulus onset (80-100 ms) and the average of the magnitude measured in a window of equal duration near the end of the offset of the contralateral noise (from 280 to $300 \mathrm{~ms}$ poststimulus onset). These same measurement windows were used to measure phase from the phase temporal waveforms. Justification for this procedure was based on the observation that the average value over $540-550 \mathrm{~ms}$ of the switched $\mathrm{fl}$ condition is similar to the average value recorded 80-100 ms for the continuous condition. The average of the envelope near the end of the DPOAE for the switched fl condition is an approximation of the response that would be recorded with quasi-continuous primaries as is more typically the case in the literature.

\section{Distortion product otoacoustic emission stimulus} frequencies and levels

Distortion product otoacoustic emission input-output functions were studied for five stimulus frequency pairs, which are indicated in Table 1 . These f2 frequencies span a range of sensitive hearing in the rat and were ones that could be recorded adequately by our equipment. The $\mathrm{I} / \mathrm{O}$ function for the highest $\mathrm{f} 2$ frequency was measured first, followed by the other f2 frequencies in descending order. The level of the f2 stimulus ranged from DPOAE threshold for that frequency to $75 \mathrm{~dB}$ SPL and was incremented in 5-dB steps. The level of $\mathrm{fl}$ was always presented $10 \mathrm{~dB}$ higher than that for f2. At each frequency, the measurement sequence started with the switched $\mathrm{fl}$ condition at L1 and L2 levels that produced the first visible response. Fifty responses were averaged and stored off-line. After that recording sequence, the f1 was changed to continuous, and a contralateral noise was introduced at $60 \mathrm{~dB} \mathrm{SPL}$ (for one rat, noise was $70 \mathrm{~dB}$ SPL). Again, 50 responses were averaged and stored. This recording sequence was repeated as L1 and L2 were raised together in 5-dB steps, until the highest L2 $=75 \mathrm{~dB}$ was reached.

After completing the entire array of measurements, the rat was transported to the surgical room and the bulla was opened. Tendons of the MEMs were cut using microscissors and electrocautery. The muscle was laid over the open bulla, or in some animals, bone wax was applied to the open bulla. The rat was then repositioned in the booth. After recalibrating the sound pressure in the ear canal, the measurements described above were repeated with the exception that DPOAEs evoked by the f2 frequencies of 12.5 and $16 \mathrm{kHz}$ were recorded last.

\section{TABLE 1}

Primary frequencies used and the corresponding 2f1-f2 DPOAE frequency

Frequencies $(\mathrm{kHz})$

\begin{tabular}{lrr}
\hline$D P O A E$ & $f 1$ & $f 2$ \\
\hline 3.6 & 4.8 & 6.0 \\
4.8 & 6.4 & 8.0 \\
6.0 & 8.0 & 10.0 \\
7.5 & 10.0 & 12.5 \\
9.6 & 12.8 & 16.0 \\
\hline
\end{tabular}


We chose to record DPOAEs evoked by the three lower f2 frequencies, as DPOAEs could be recorded over a wider stimulus range than those evoked by the two highest f2 frequencies. During the second half of the recording period, fluid slowly accumulated in the middle-ear cavity in almost every animal. If DPOAE levels continually decreased, the animal was taken back to the surgery room to suction the fluid. After repositioning and recalibration in the recording booth, DPOAE levels returned to baseline levels. In five rats, I/O functions for all five DPOAE frequencies were recorded both before and after sectioning of the middle-ear muscles. In two rats, only partial data were obtained after sectioning the middle-ear muscles because fluid accumulation was too rapid. For an additional two rats, no data were recorded after sectioning the middle-ear muscles because DPOAE amplitude had decreased by more than 15 $\mathrm{dB}$ or were completely absent. In these rats, the stapedial artery had been nicked during middle-ear muscle section, and it was felt that possible cochlear damage occurred.

Except for the results shown in Figure 12, the level of $\mathrm{f} 2$ was always $10 \mathrm{~dB}$ below the level of $\mathrm{f} 1$. An experiment was undertaken to investigate whether the effects of varying the level of $\mathrm{f} 2$ relative to $\mathrm{fl}$ in 1-dB increment in the rat varied in a similar manner to that reported in guinea pigs (Maison and Liberman 2000). Responses in two rats were studied for this purpose.

\section{Calibration}

The "constant voltage" method was used to set the intensity of the primary stimuli. Calibrations of both primary channels were performed at $1.0 \mathrm{kHz}$ in the ear of the subject, and intensity was assumed to remain constant with stimulus frequency. This method has been shown to be more accurate than attempting to calibrate the stimuli using the recording microphone (Siegel and Hirohata 1994).

Careful examination of responses in a coupler and computer simulations were used to insure that no responses reported here were "artifacts" resulting from the gating of the primary stimuli. For the stimulus conditions reported here, all onset and offset transients associated with the primary stimuli were at least $80 \mathrm{~dB}$ below the level of the $\mathrm{fl}$ stimulus. Additionally, the noise floor measured before stimulus onset was typically $-5 \mathrm{~dB}$ SPL or lower.

\section{RESULTS}

Distortion product otoacoustic emission temporal responses for magnitude and phase showing $\Delta \mathrm{LI}$ (switched f1 stimulus timing) in one rat are shown in Figure 3 . The f2 frequency was $10 \mathrm{kHz}$. The temporal envelope magnitudes are shown in the top row, and the corresponding phases are shown in the bottom row. Each panel, moving from left to right, shows DPOAE responses evoked by increasingly higher primary levels in 5-dB steps. The DPOAE temporal envelopes for magnitude are similar to those found by other researchers in various species (Liberman et al. 1996; Kim et al. 2001; McGee et al. 2000; Sun and Kim 1999). The DPOAE magnitude near the onset initially increased with intensity, decreased to a minimum around L2 $=70 \mathrm{~dB}$ SPL, then substantially increased with intensity at the highest primary levels. If one were to plot the I/O function, a "notch" would be observed at $70 \mathrm{~dB}$ SPL. Subsequently, $\Delta \mathrm{LI}$ (the difference between the DPOAE magnitude at the onset and that near the end of the trace) initially increases with intensity, then decreases and reverses direction (i.e., becomes negative), then increases again.

Temporal envelopes for phase also change with time and, in a manner, linked with magnitude changes. Near the onset of the stimulus, the phase changes rapidly by small amount, then there are small, slow changes during the later duration of the stimuli. The direction of phase change is linked with the direction of the magnitude change. In the quasisteady-state part of the response, the phase increases slightly and slowly with time when the corresponding DPOAE magnitude decreases with time. When the DPOAE onset magnitude increased with time (L2 = $70 \mathrm{~dB}$ SPL), the corresponding phase decreased with time. At the highest intensity, phase remained as decreasing with time.

The $\Delta \mathrm{LC}$ (measured using the $\mathrm{fl}$ continuous timing) for the same rat and primary stimulus conditions is shown in Figure 4. The format of the figure is similar to that used in Figure 3. The change in magnitude and phase from approximately 120 to $320 \mathrm{~ms}$ is a result of the introduction of the $60 \mathrm{~dB}$ SPL noise presented contralaterally. The amount of $\Delta \mathrm{LC}$ increases, then decreases, and then reverses absolute value (becomes positive) at the highest intensity level. The change in $\Delta \mathrm{LC}$ and its reversal in absolute value exhibits a similar pattern seen for $\Delta$ LI. The phase behavior changes in a similar way as well. That is, during contralateral stimulation when magnitude decreased, $\triangle \mathrm{PC}$ was positive (phase became less negative). The $\triangle \mathrm{PC}$ reversed direction when the DPOAE magnitude increased during presentation of the contralateral noise. In both Figures 3 and 4 , it appears that the starting DPOAE phase exhibits a pattern of small, increasing phase with f2 level with a large jump in phase near the notch.

Distortion product otoacoustic emission temporal envelopes for $\Delta \mathrm{LI}$ and $\Delta \mathrm{LC}$ in the same rat after 


\section{F2 $=10.0 \mathrm{kHz}$, rat072701}
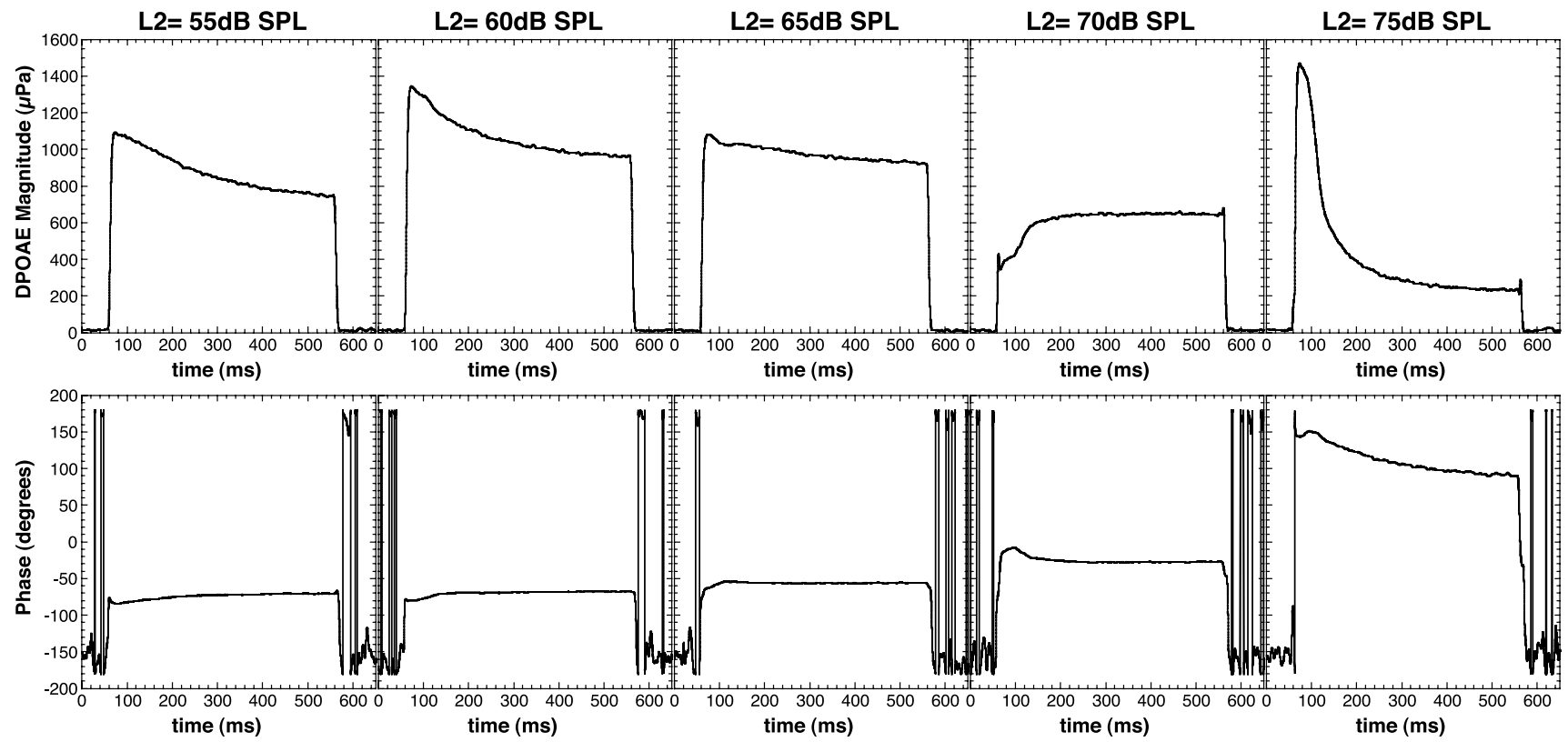

FIG. 3. DPOAE magnitude (top row) and phase (bottom row) using the switched $\mathrm{f} 1$ condition and an $\mathrm{f} 2$ frequency of $10 \mathrm{kHz}$ in rat 072701. Middle-ear muscles were intact. The f2 stimulus levels used to evoke the magnitude and phase temporal responses are indicated at the top of each magnitude panel. $\Delta \mathrm{LI}$ changes with primary level and reverses direction for $\mathrm{L} 2=70 \mathrm{~dB}$ SPL. $\Delta \mathrm{PI}$ are small and exhibit a pattern that is tied with the stimulus level.

F2=10.0kHz, rat072701
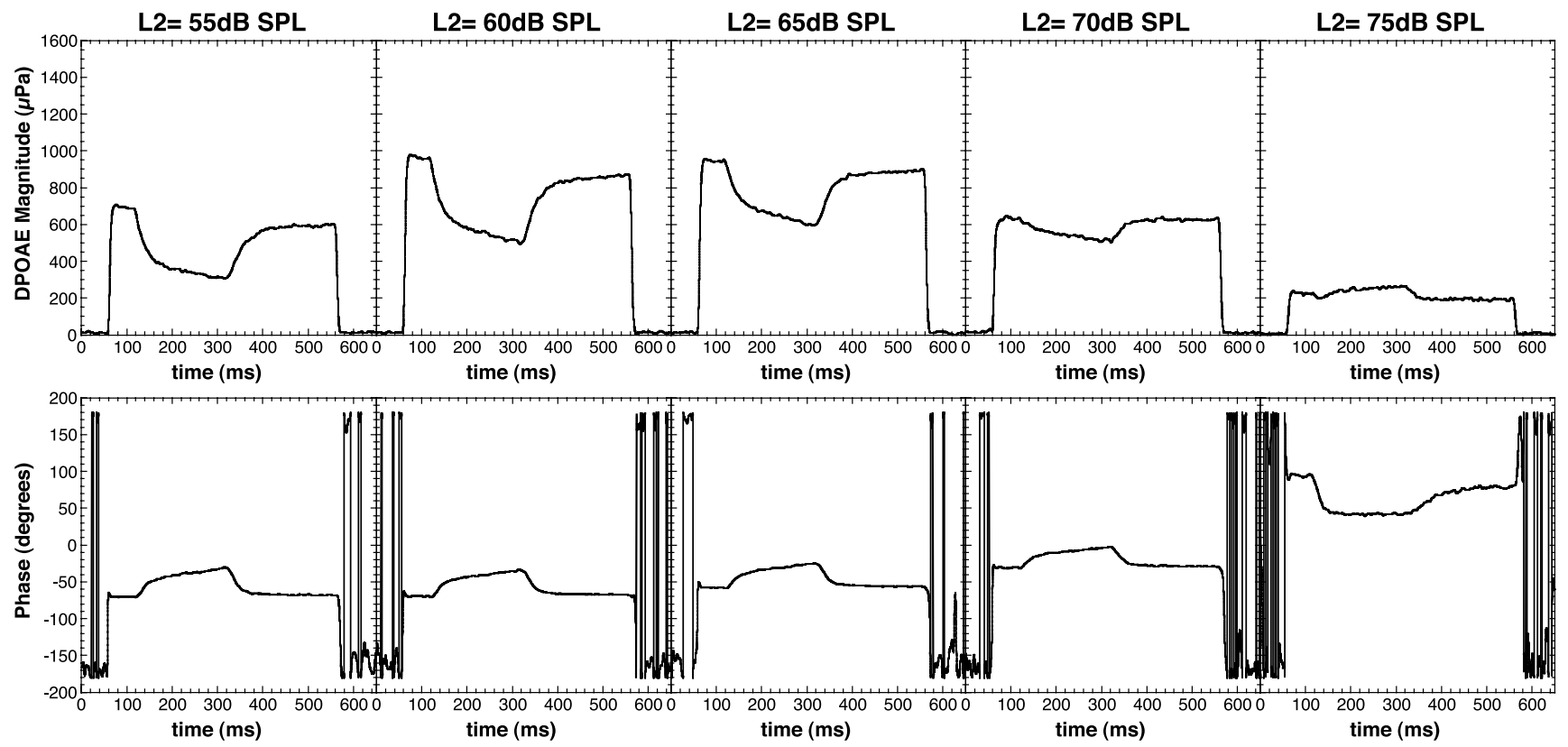

FIG. 4. DPOAE magnitude (top row) and phase (bottom row) using the continuous $\mathrm{f} 1$ condition for an $\mathrm{f} 2$ frequency of $10 \mathrm{kHz}$ in the same rat whose data are shown in Figure 3. $\Delta \mathrm{LC}$ changes with stimulus level, becomes small for $\mathrm{L} 2=70 \mathrm{~dB} \mathrm{SPL}$, and changes direction for $\mathrm{L} 2=75 \mathrm{~dB}$ SPL. $\triangle \mathrm{PC}$ changes are linked with stimulus levels as well. 
cutting both the middle-ear muscles are illustrated in Figures 5 and 6 , respectively. It can be seen that both $\Delta \mathrm{LI}$ and $\Delta \mathrm{LC}$ are reduced considerably after sectioning of the middle-ear muscles. When residual $\Delta \mathrm{LI}$ and/or $\Delta$ LC were observed, corresponding phase changes were either absent or very small (Figure 6, $\mathrm{L} 2=75 \mathrm{~dB} \mathrm{SPL}$ ). It is presumed that the residual magnitude changes are the result of activation of the MOC reflex. Although smaller in amplitude, the time courses of the residual magnitude changes appear similar to those seen when the MEMs are intact.

Figure 7 shows DPOAE I/O functions using the switched $\mathrm{fl}$ stimulus paradigm. The left panel illustrates the $\mathrm{I} / \mathrm{O}$ function before MEM sectioning, and the middle column depicts data after MEM sectioning. The solid lines illustrate the $\mathrm{I} / \mathrm{O}$ function using the DPOAE level computed from the onset of the trace (first hatched area shown in panel 2a), and the dashed line depicts the $\mathrm{I} / \mathrm{O}$ function computed from the end of the temporal response (second hatched area in panel 2a). Each row is for a different f2 frequency. Before MEM section, several observations can be made. First, the I/O functions are different depending on f2 frequency. Secondly, the I/O functions for the onset and the end of the trace are different from each other. For all but the lowest frequency, the onset and offset levels are similar at low stimulus levels, but as stimulus level is raised, the DPOAE levels at the two measurement periods are different. With increasing intensity, where there are nonmonotonicities, the pattern in the I/O function for the end of the trace appears to be shifted to the right. The marked differences in $\mathrm{I} / \mathrm{O}$ functions between the beginning and end of the trace are much reduced after MEM section, as can be seen in the middle column. There are still small differences between the I/O functions, but they are quite similar, even with respect to nonmonotonicities. These findings suggest that the large differences in the $\mathrm{I} / \mathrm{O}$ functions between the beginning and end of the temporal responses as shown in the left panel are due mainly to middle-ear muscles. The panel at the right shows a comparison in onset $\mathrm{I} / \mathrm{O}$ functions before (thicker line) and after MEM sectioning (thinner line). One important finding is that after MEM section, DPOAE levels are reduced and not in the same manner for each frequency. The majority of functions are similar to f2 frequencies of $6,8,12.5$, and $16 \mathrm{kHz}$, where there is a shift of the function to the right and/or decreased levels in areas of saturation. Few differences were like the function for $\mathrm{f} 2=$ $10 \mathrm{kHz}$, where low levels are changed and not high levels. In all cases, slopes of the $\mathrm{I} / \mathrm{O}$ functions remain similar pre- and post-MEM section. Even after MEM section, notches still exist, but not always at the same stimulus levels as before MEM sectioning. Because of the nonmonotonic $\mathrm{I} / \mathrm{O}$ functions, both before and after MEM sectioning, it is potentially misleading to consider the effects of cutting MEMs at any one primary level. The difficulty is compounded by the shifts in the $\mathrm{I} / \mathrm{O}$ function that were observed after sectioning the muscles. The differences in the $\mathrm{I} / \mathrm{O}$

F2=10.0kHz, rat072701
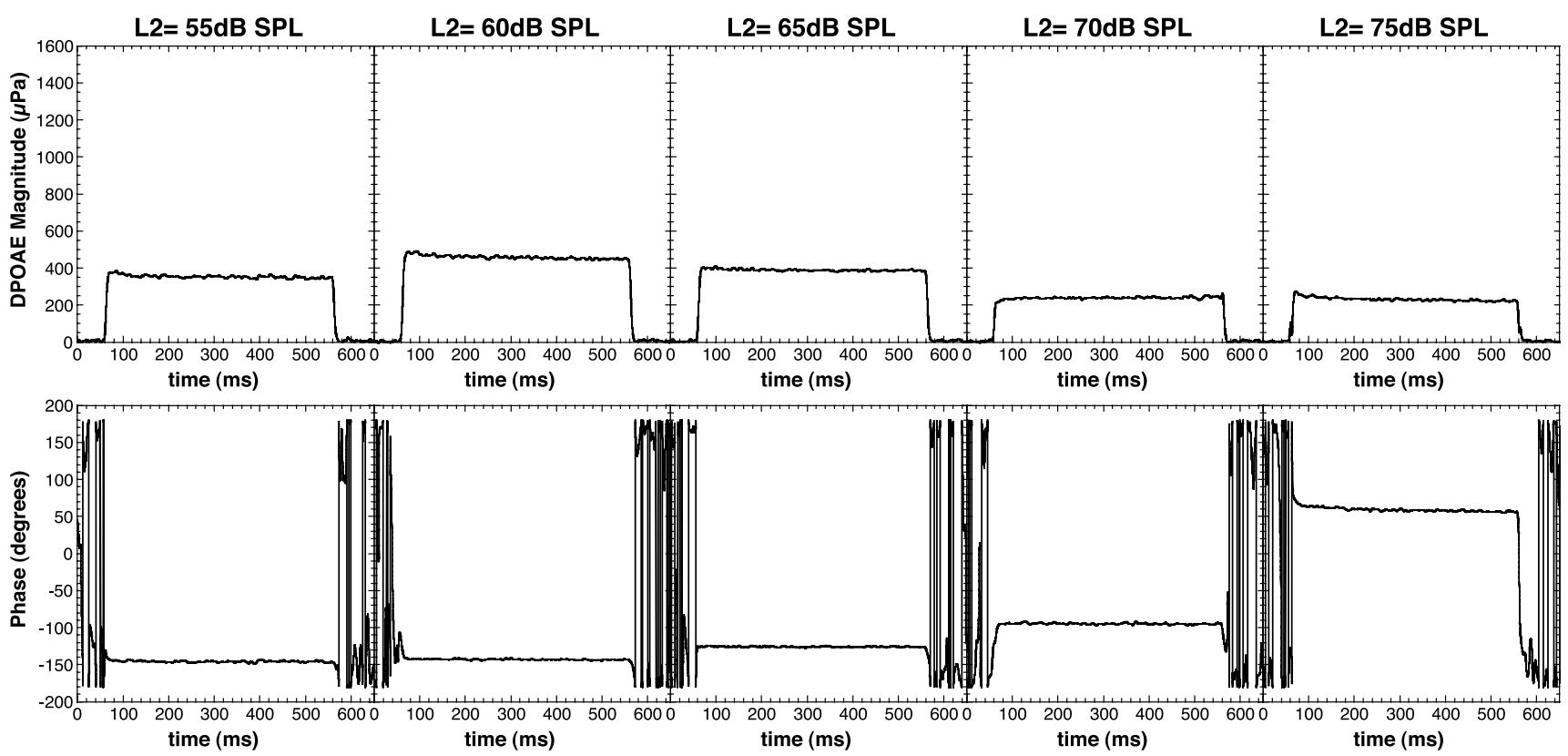

FIG. 5. DPOAE magnitude (top row) and phase (bottom row) using the switched f1 condition for the same rat whose data are shown in Figures 3 and 4 after sectioning of the MEMs. $\Delta \mathrm{LI}$ and $\Delta \mathrm{PI}$ are substantially reduced after MEM sectioning. 
F2=10.0kHz, rat072701
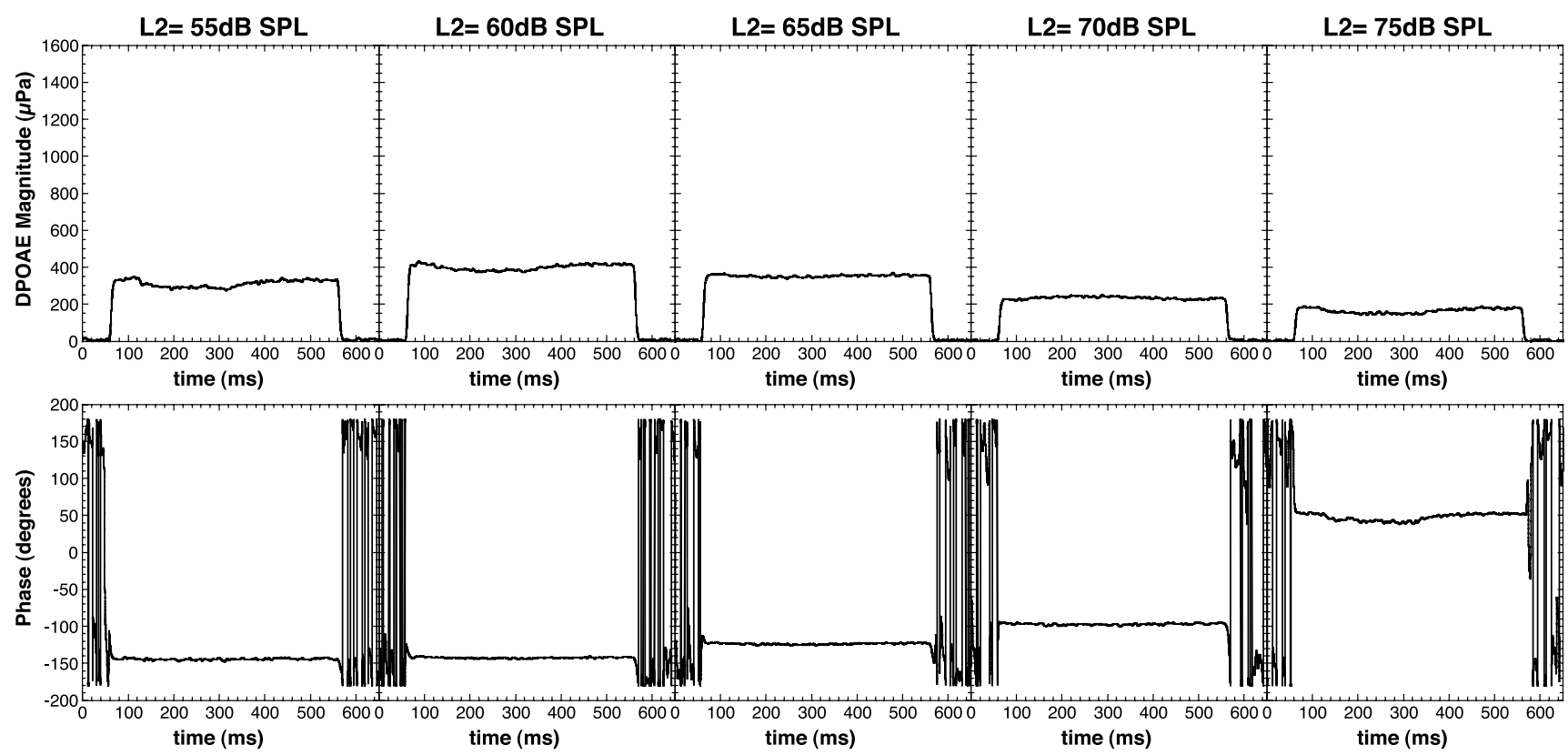

FIG. 6. DPOAE magnitude and phase using the continuous $\mathrm{f} 1$ condition in rat 072701 after sectioning of the MEMs. $\triangle \mathrm{LC}$ and $\triangle \mathrm{PC}$ are substantially reduced.

functions pre- and post-MEM section were attributed to the accumulation of fluid in the middle-ear space and the change in bulla integrity after MEM section.

The amounts of $\Delta \mathrm{LI}$ and $\Delta \mathrm{LC}$, before and after sectioning of the MEMs in three rats, are shown in Figures 8 and 9, respectively. Each column illustrates data for a different rat. Each panel shows either $\Delta \mathrm{LI}$ (Fig. 8) or $\Delta$ LC (Fig. 9) for the five different primary frequencies studied, with the lowest-frequency primaries in the top panels and increasing frequency with successively lower panels. The dotted or gray bars represent $\Delta \mathrm{LI}$ or $\Delta \mathrm{LC}$ measured with intact MEMs, and the black bars represent these phenomena after the MEMs had been cut. Several trends are apparent for both figures. First, the absolute value of $\Delta \mathrm{LI}$ or $\Delta \mathrm{LC}$ is greater than $10 \mathrm{~dB}$ and, occasionally, greater than $20 \mathrm{~dB}$ for some $\mathrm{f2}$ intensities and DPOAE frequencies prior to MEM sectioning. Second, the amount of $\Delta \mathrm{LI}$ or $\Delta \mathrm{LC}$ is smaller for higher frequencies. Third, for all stimulus conditions, both $\triangle \mathrm{LI}$ and $\triangle \mathrm{LC}$ are reduced after sectioning the MEMs and rarely do the measures exceed $5 \mathrm{~dB}$. We interpret these affects as a result of the MOC reflex. These observations support the conclusion that $\Delta \mathrm{LI}$ and $\triangle \mathrm{LC}$ in the rat are mediated, for the most part, by the MEM reflex. Fourth, there is variability among individuals in the size of $\Delta \mathrm{LI}$ and $\Delta \mathrm{LC}$, both before and after MEM sectioning. Fifth, the magnitude of $\Delta \mathrm{LC}$ is greater than $\Delta \mathrm{LI}$. Finally, the amount of either $\Delta \mathrm{LI}$ or $\Delta \mathrm{LC}$, as well as whether it is a reduction in DPOAE level or an enhancement, is highly depen- dent on the $\mathrm{I} / \mathrm{O}$ function. This is true for before and after MEM sectioning and can be understood best by the data illustrated in Figure 10.

The relationship between amount of $\Delta \mathrm{LI} / \Delta \mathrm{LC}$ and the $\mathrm{I} / \mathrm{O}$ function is illustrated in Figure 10. Input-output functions for a DPOAE measured at $\mathrm{f} 2=6 \mathrm{kHz}$ before MEM sectioning were measured near stimulus onset for the switched fl condition (black line) and the continuous fl condition (dashed line). The vertical bars show the amount of $\Delta \mathrm{LI}$ (black bars) and $\triangle \mathrm{LC}$ (striped bars) at each intensity. The I/O functions are nonmonotonic, each with a pronounced notch at a mid-to-high intensity. The data plotted here are representative of the entire data set. The direction and extent of $\Delta \mathrm{LI}$ and $\Delta \mathrm{LC}$ are related to the slope of the DPOAE I/O function. The direction of the $\Delta \mathrm{LI}$, decreasing or increasing, generally depends on whether the slope of the input/output function is positive or negative, respectively. From the maximum peak of the $\mathrm{I} / \mathrm{O}$ function to the minimum of the notch, where the slope is negative, is where apparently paradoxical increasing $\Delta \mathrm{LI}$ (and increasing responses to contralateral noise) is observed. At the minimum, notch response shape can be multiphasic because of the discontinuity in the slope.

Distortion product otoacoustic emission phase from three rats is illustrated in Figure 11. All data are for the continuous $\mathrm{fl}$ condition as it was easier to measure phase more reliably from this condition as compared with the switched fl condition. Absolute 

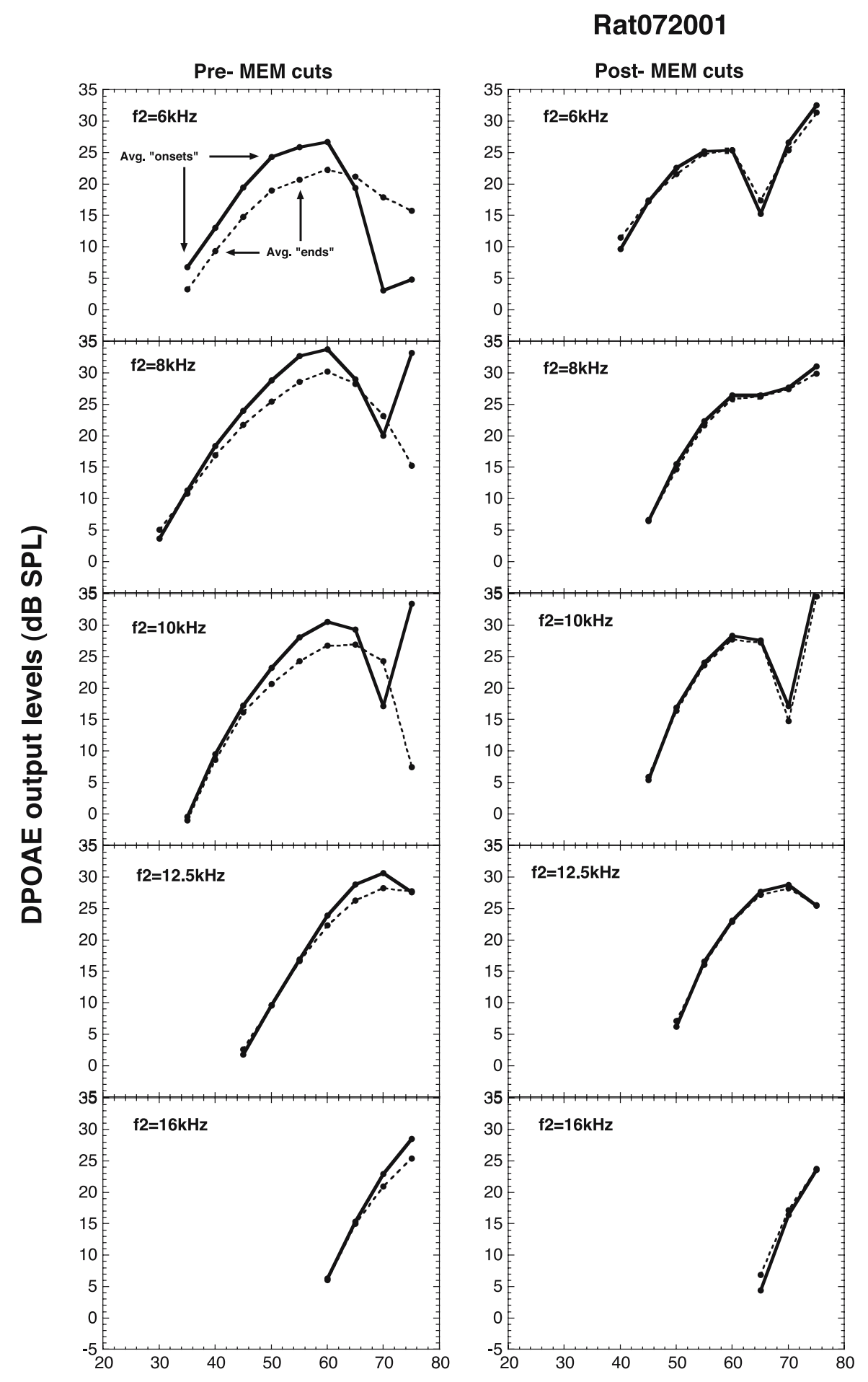

\section{Onset Avgs. Pre+Post MEM cuts}

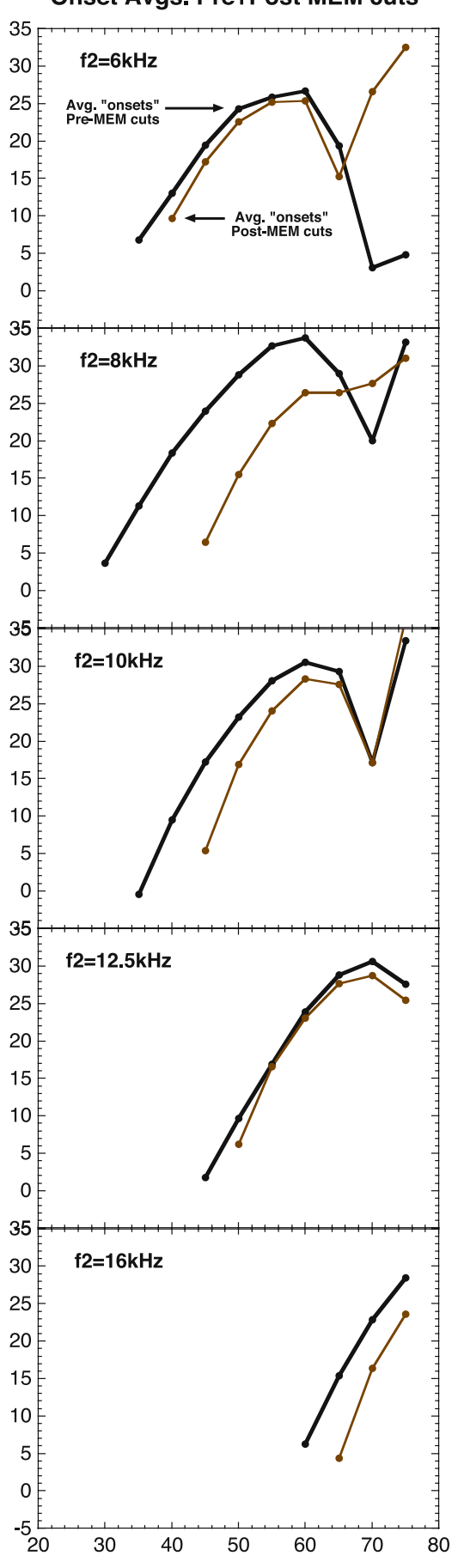

\section{L2 input (dB SPL)}

FIG. 7. DPOAE $\mathrm{I} / \mathrm{O}$ functions for one rat using the $\mathrm{f} 1 \mathrm{switched}$ condition. I/O functions are shown before MEM section (left column), after MEM section (middle column), and comparison before and after MEM section (right column). Each panel is a different $\mathrm{f} 2$ frequency. Solid and dotted lines depict DPOAE levels at the beginning and end of the traces, respectively (time periods shown by hatched lines in Figure 2), in the left and middle columns. In the right column, solid and dashed lines represent pre- and postMEM section, respectively. 

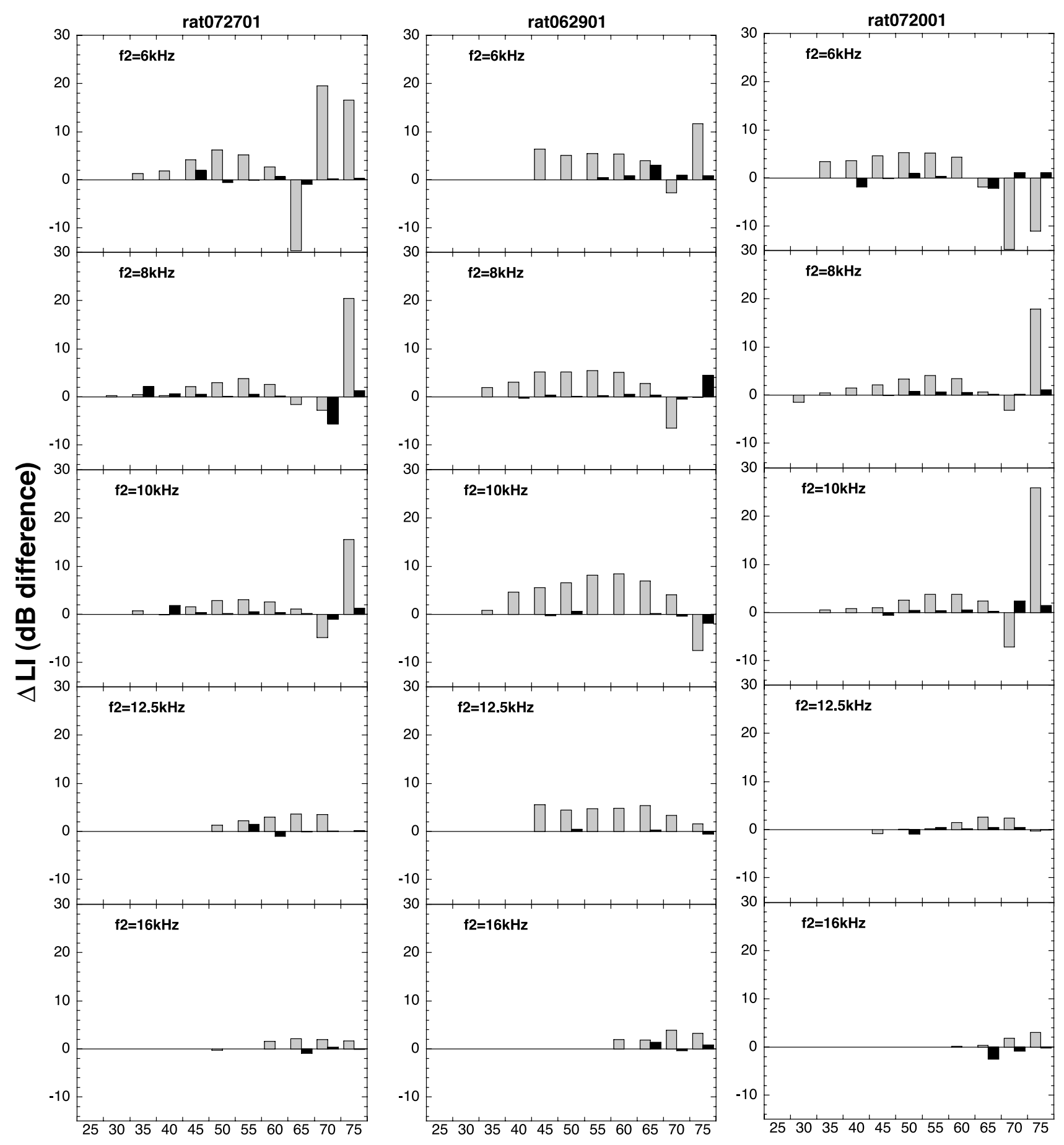

\section{L2 (dB SPL)}

FIG. 8. $\Delta \mathrm{LI}$ as a function $\mathrm{L} 2$ for three rats. Each column illustrates data for a different rat. Each row depicts data for different $\mathrm{f} 2 \mathrm{frequencies,}$ starting from the lowest frequency tested at the top of the column to the highest frequency tested at the bottom of the column. Textured bars represent $\Delta \mathrm{LI}$ when MEMs were intact, and black bars depict $\Delta \mathrm{LI}$ after MEM sectioning.

phase was not calibrated, but the relative phases within an I/O are valid. The DPOAE phase before sectioning the MEMs is depicted by closed circles, and the DPOAE phase near the offset of the contralaterally presented noise is depicted by open triangles.
Distortion product otoacoustic emission phase after MEM sectioning is depicted by closed squares, and DPOAE phase near the offset of the contralaterally presented noise after MEM sectioning is depicted by open diamonds. Solid arrows and dashed arrows 

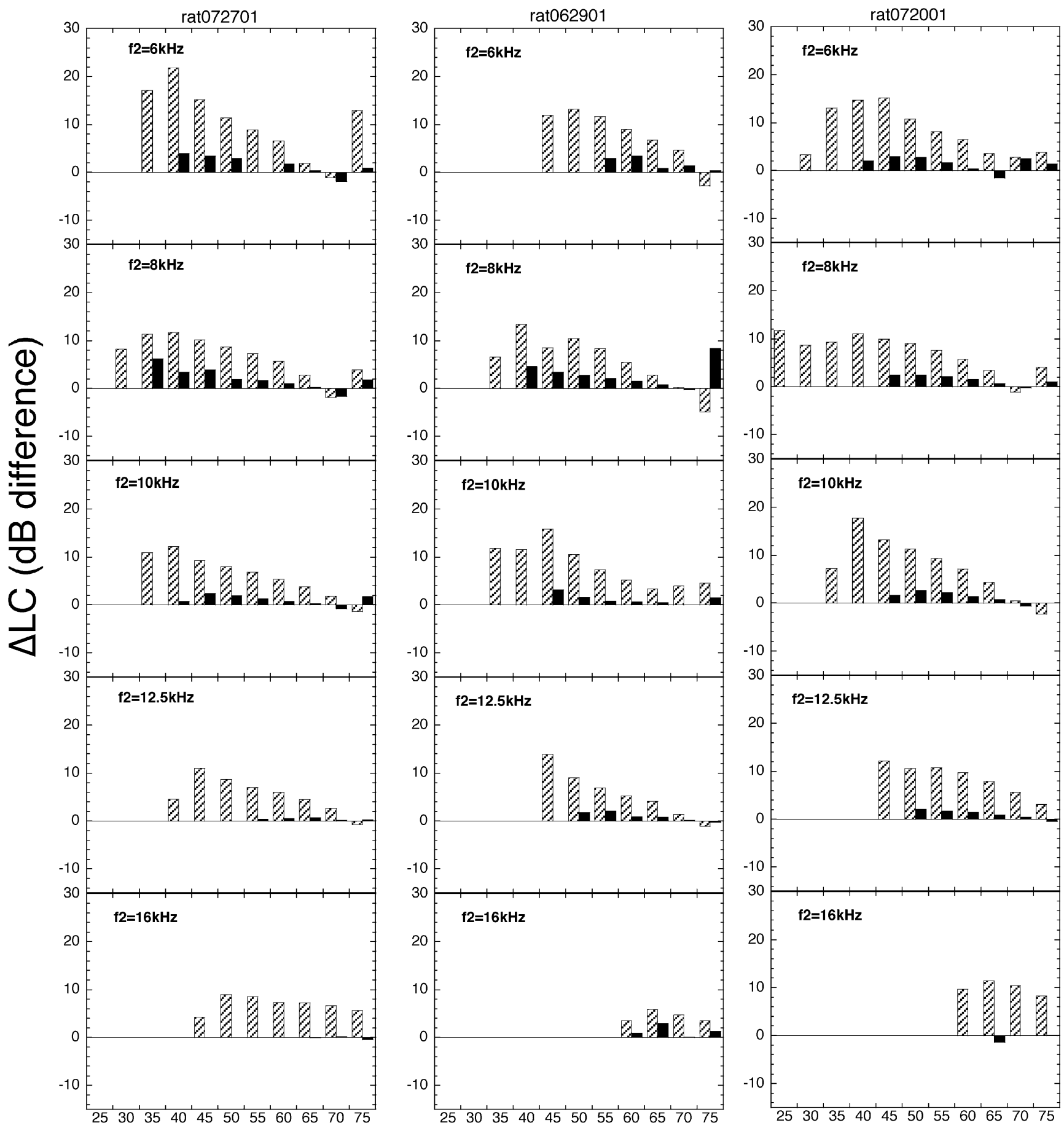

\section{L2 (dB SPL)}

FIG. 9. $\Delta \mathrm{LC}$ as a function of $\mathrm{L} 2$ level for three rats. The same plotting conventions were the same as those used for Figure 8.

indicate the levels at which a "notch" or low point in the I/O function (found at the highest primary levels we presented) was found pre- and post-MEM section, respectively. The top panel (a) shows data representative of rats having notched I/O functions. Distortion product otoacoustic emission phase before MEM and after MEM sectioning showed small changes in phase with increasing signal level. Between L2 levels of 70 and $75 \mathrm{~dB}$ SPL, there were large changes in phase, approximately $180^{\circ}$. The $\mathrm{I} / \mathrm{O}$ function preMEM section exhibited a sharp decrease at $75 \mathrm{~dB}$ SPL and a "notch" at $70 \mathrm{~dB}$ SPL post-MEM section. The large phase difference occurred near stimulus levels at which there was a notch in the $\mathrm{I} / \mathrm{O}$ function. As 


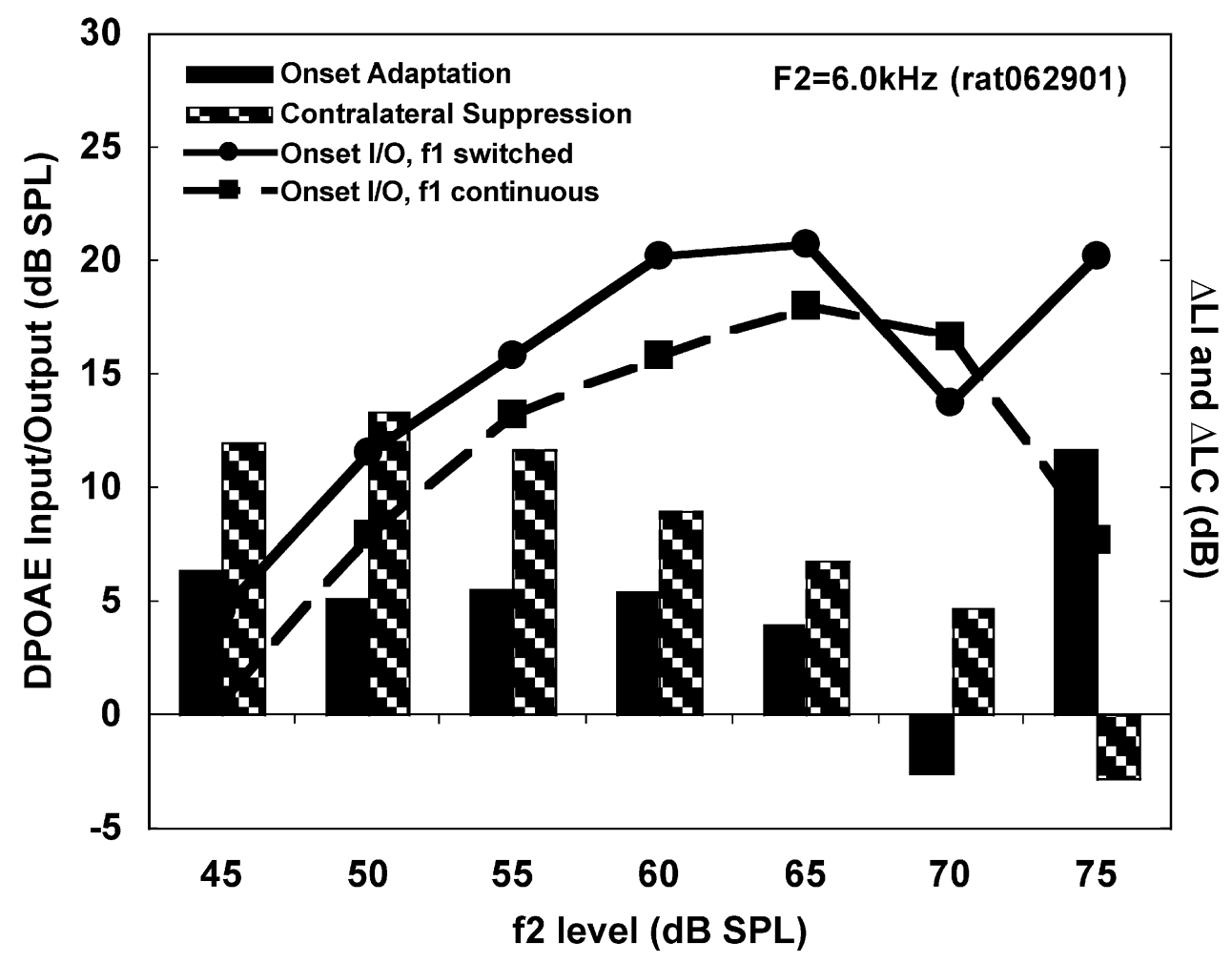

FIG. 10. DPOAE $\mathrm{I} / \mathrm{O}$ functions are plotted as a function of $\mathrm{f} 2$ level using the $\mathrm{f} 1$ switched condition (solid lines) and the $\mathrm{f} 1 \mathrm{continuous} \mathrm{condition}$ (dashed lines) pre-MEM section. For each $\mathrm{f} 2$ level, the $\Delta \mathrm{LI}$ (black bars) and $\Delta \mathrm{LC}$ (patterned bars) are plotted. It can be seen that the direction of $\Delta \mathrm{LI}$ and $\Delta \mathrm{LC}$ are tied with the nonmonotonicities of the $\mathrm{I} / \mathrm{O}$ functions.

shown by the open triangles, the DPOAE measured during the contralateral noise is fairly flat for L2 levels less than $75 \mathrm{~dB}$ SPL. There is a phase lead during presentation of contralateral noise pre-MEM section below the I/O function notch. After MEM section, there is no difference in DPOAE phase during contralateral stimulation, except for a small phase lag of approximately $10^{\circ}$ at the highest primary level, which was close to where the notch of the I/O function occurred. These data illustrate the findings for almost all of our data. For $\triangle \mathrm{PC}$, small (on the order of $10-20^{\circ}$ ) phase lags were noticed in the region of an $\mathrm{I} / \mathrm{O}$ notch. In only two instances was this not true, and one example is shown in panel b. Data are similar to those shown in panels a, with the exception that a phase lead of approximately $25^{\circ}$ was seen at the notch of the $\mathrm{I} / \mathrm{O}$ function during the presentation of contralateral noise after MEM section. This is our only example of a $\triangle \mathrm{PC}$ that had a phase lead, rather than a phase lag, during contralateral noise stimulation post-MEM section. Additionally, there was no measurable $\Delta \mathrm{LC}$, only $\Delta \mathrm{PC}$. Panel $\mathrm{c}$ (lowest panel) illustrates data from another animal at the same frequency as that shown in panel a. For this animal, there was no notch in the $\mathrm{I} / \mathrm{O}$ function preMEM section. Only small phase changes were recorded with increasing L2 level. After MEM section,
DPOAE levels were low at $75 \mathrm{~dB}$ SPL, and the approximate $180^{\circ}$ phase change is noted. All our data followed these examples in panels a and c, except for the one shown in panel $b$ and one other trace where $a$ $\triangle \mathrm{PC}$ of $10^{\circ}$ was noted a low set of primary levels where there was no notch in the $\mathrm{I} / \mathrm{O}$ function. $\Delta \mathrm{PI}$ were similar to $\triangle \mathrm{PC}$ in that no changes were noted for most of the functions after MEM section. A few, small $\Delta$ PI (phase lags) post-MEM sections were noticeable and only occurred in the region of the I/O notch.

In two rats, we have observed $\Delta \mathrm{LI}$ and $\Delta \mathrm{PI}$ in the vicinity of $\mathrm{I} / \mathrm{O}$ function notch in great detail, following similar procedures to that of Maison and Liberman (2000) and Kujawa and Liberman (2001). In these studies, it was shown that with a fixed $\mathrm{f} 1$ level, and f2 level increasing in 1-dB steps starting from $15 \mathrm{~dB}$ below the level of $\mathrm{f} 1$, onset adaptation progressed from a positive to a negative value. In addition, there was a large amount of onset adaptation near the primary level that the effect switched from positive to negative. Kujawa and Liberman (2001) noted that this effect was seen at the DPOAE $\mathrm{I} / \mathrm{O}$ function notch. Our goal was to see if responses in the rat, for which MEMs appear to be a major contributor to DPOAE reduction, were similar to those in the guinea pig, in which changes appear to be mediated by the MOC reflex. In the current study, 


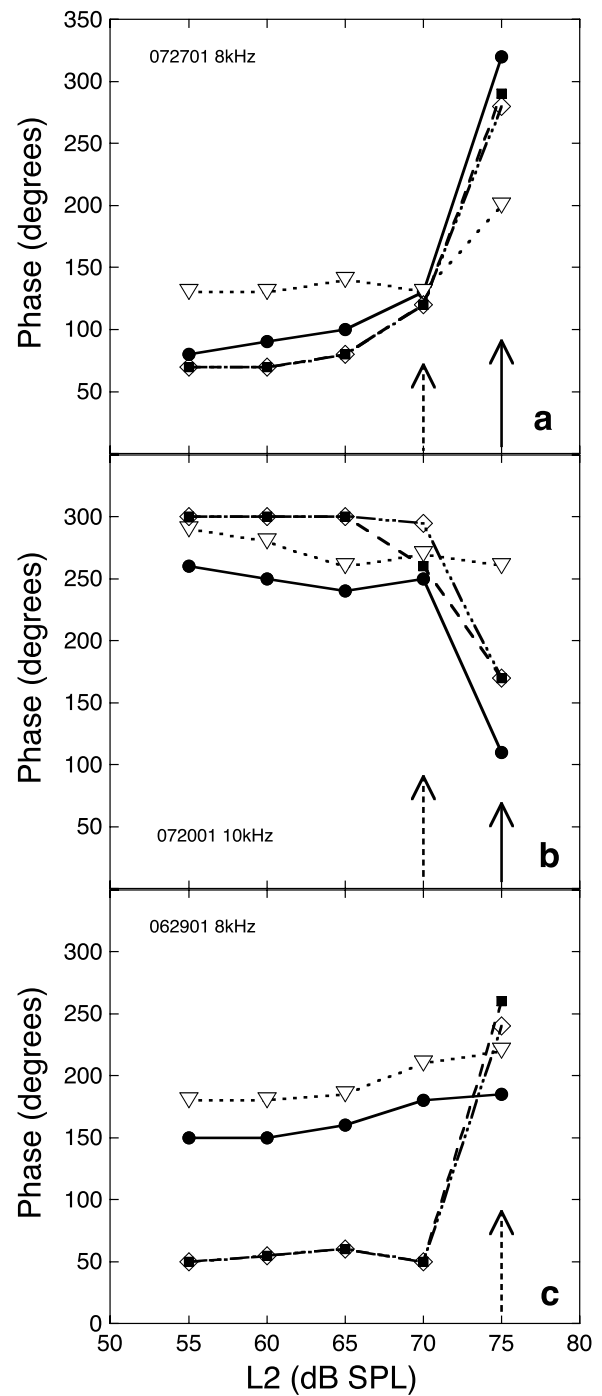

FIG. 11. DPOAE phase as a function of $L 2$ level for the $\mathrm{f1}$ continuous condition in three rats. Circles represent DPOAE phase before MEM section. The closed symbols are DPOAE phase at the beginning of the trace, and open symbols are DPOAE phase near the end of the time frame that contralateral noise was presented. Closed squares represent DPOAE phase at the beginning of the trace after MEM section, and open diamonds represent DPOAE phase near the end of the time frame that contralateral noise was presented. Solid and dashed arrows indicate the level at which there was a "notch" in the $\mathrm{I} / \mathrm{O}$ function or low DPOAE level at the highest primary level pre-MEM and post-MEM sectioning, respectively. Each panel represents data from a different rat.

the intensity of $\mathrm{fl}$ was fixed at the level that produced the local minimum in the magnitude $\mathrm{I} / \mathrm{O}$ function. The intensity of $\mathrm{f2}$ was varied in 1-dB steps from a value $15 \mathrm{~dB}$ below that of $\mathrm{f} 1$ to a value that was $5 \mathrm{~dB}$ greater. Results for one rat are shown in Figure 12 with magnitude being displayed in the left panel and the corresponding phase in the right panel. To compare DPOAE changes with level more clearly, each trace has been normalized to its own steady-state portion at the end of the trace. Both the magnitude and phase temporal responses exhibit nonmonotonic, complex waveforms that change dramatically with 1-dB changes in $\mathrm{f} 2$ level. For f2 levels between 71 and $74 \mathrm{~dB}$ SPL, the initial waveform is positive, followed by a negative peak that becomes more negative with increasing SPL. When $\mathrm{f} 2=75 \mathrm{~dB}$ SPL, the negative peak disappears, and the waveform looks as if it is decreasing $\Delta \mathrm{LI}$. The phase function shows the usual relationship to magnitude; that is, it is a decreasing function when magnitude is increasing. However, it, too, has a complicated waveform. It reverses its sign, similar to that of the magnitude temporal response function, except that it changes direction between f2 levels of 72 and $73 \mathrm{~dB}$ SPL, and the starting phase shifts approximately $180^{\circ}$.

\section{DISCUSSION}

Whereas it has been established convincingly in some species that $\triangle \mathrm{LI}$ and $\Delta \mathrm{LC}$ result from activation of the MOC reflex by either cutting the MEMs (Liberman et al. 1996; Puel and Rebillard 1990) or cutting the OCB (Kujawa and Liberman 2001), data from the rat suggest that $\Delta \mathrm{LI}$ and $\Delta \mathrm{LC}$ can be attributed largely to MEM reflexes. Across species, the most common range and/or mean of $\Delta \mathrm{LI}$ and $\Delta \mathrm{LC}$ tends to be between 0.5 and $4 \mathrm{~dB}$. However, some very large effects, as much as $20 \mathrm{~dB}$, have been reported (McGee et al. 2000; Kujawa and Liberman 2001; Maison and Liberman 2000). In the rat, $\Delta$ LI ranged from $-14 \mathrm{~dB}$ to as high as $26 \mathrm{~dB}$, similar to the large effects reported in guinea pigs, in which it has been demonstrated that the changes are due to the MOC (e.g., Kujawa and Liberman 2001). Data from one rabbit, in which it has been interpreted that $\Delta \mathrm{LI}$ is influenced by MEMRs, demonstrated a $\Delta \mathrm{LI}$ of approximately $8 \mathrm{~dB}$. One reason why it is difficult to compare absolute values of $\Delta \mathrm{LI}$ and $\Delta \mathrm{LC}$ across species, and even across animals, is because the size and direction of change are heavily dependent on the $\mathrm{I} / \mathrm{O}$ function. Moreover, in the notch region, $\Delta \mathrm{LI}$ can be extremely large with differences in stimulus level (Maison and Liberman 2000; McGee et al. 2000; Kujawa and Liberman 2001). Without knowing the underlying $\mathrm{I} / \mathrm{O}$ function, it is difficult to compare the magnitudes of $\Delta \mathrm{LI}$ and $\Delta \mathrm{LC}$ across animals and species. Furthermore, looking at DPOAE magnitude only, especially at one primary level, will not allow us to adequately quantify the effects. In sum, one cannot determine from size of $\Delta \mathrm{LI}$ or $\Delta \mathrm{LC}$ whether the effect is due to MOC or MEMRs. In other words, it is the case that $\Delta \mathrm{LI}$ and $\Delta \mathrm{LC}$ across species appear to be similar phenomena, yet these phenomena 

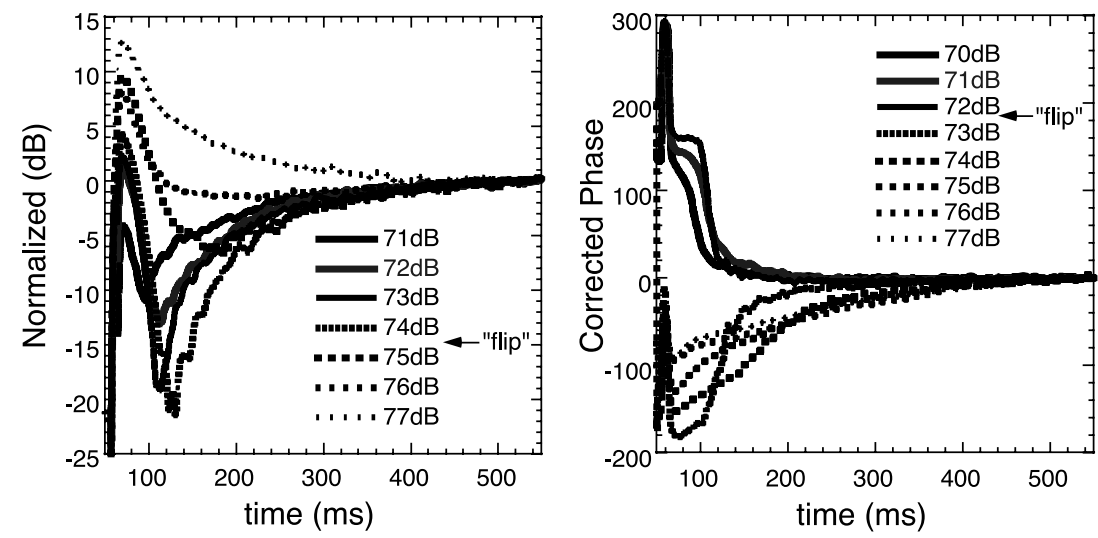

FIG. 12. DPOAE magnitude (left panel) and phase (right panel) measured in one rat. The f1 level was fixed at the level that produced the local minimum in the $1 / O$ function, and the $\mathrm{f} 2$ level was varied in 1-dB steps. The shape of the waveform changes dramatically as f2 level is varied. result from different neural and effector pathways across species.

Our conclusions that MEM reflexes are the main contributor to $\Delta \mathrm{LI}$ and $\Delta \mathrm{LC}$ in the rat are similar to those reached by researchers studying awake rabbits (Luebke 2002; Whitehead et al. 1991). These researchers did not cut MEMs or cut MOC at midline, but rather used acoustic middle-ear impedance and changes in primary stimulus $\mathrm{dB}$ SPL to determine whether MEM reflexes were being activated at same levels used to measure MOC effects. They could not separate the MEM reflex and MOC reflex effects, nor show conclusively that an MOC effect was present. They concluded that $\Delta \mathrm{LI}$ and $\triangle \mathrm{LC}$ were combined MEM/MOC reflexes. Our data, along with theirs, suggest that there may be a species component to the contribution of MOC and MEMR on the reduction of DPOAEs.

One striking aspect of rat data from the current study is that there is MEMR influence at lower levels and over a broader frequency range in that rat than is typical for other species. In this respect, our data are similar to those found in albino Wistar rats. Murata et al. (1986) found that the most sensitive mean frequency that elicited the MEMR was at $3.3 \mathrm{kHz}$ $(\mathrm{SD}=1 \mathrm{kHz})$, and that the mean threshold measured through EMG recordings in either the tensor tympani or stapedius muscle was $57 \mathrm{~dB}$ SPL $(\mathrm{SD}=8 \mathrm{~dB})$. They presented data from one rat using visual inspection of muscle contraction that indicated that the threshold was approximately $47 \mathrm{~dB}$ SPL (their Figure $3 \mathrm{~b}$ ). Their group also found that thresholds were similar between stapedius and tensor tympani reflexes and between ipsilateral and contralateral reflexes. In the Wistar rat, middle-ear transmission was affected from 1 to $20 \mathrm{kHz}$.

Examples of what we interpret as MEMR influence can be seen throughout our data and are consistent with observations reported by Murata et al. (1986). For example, the $\mathrm{I} / \mathrm{O}$ functions for the lowest frequency in the left panel of Figure 7 suggest that the MEMR is activated with an $\mathrm{fl}$ frequency of $4.8 \mathrm{kHz}$ at $45 \mathrm{~dB}$ SPL (f2 level and frequency are displayed in the figure). Whereas this seems low for MEMR activation in most species, $\mathrm{fl}$ is close to the most sensitive MEMR frequency, and the level is within two standard deviations of the mean threshold for MEMR activation based on data from Murata et al. (1986). For higher frequencies, the $\mathrm{I} / \mathrm{O}$ for the end of the trace starts to diverge from the onset I/O between 40 and $80 \mathrm{~dB}$ SPL for $\mathrm{f} 1$ frequencies of $6.4-12.8 \mathrm{kHz}$ with increasingly higher levels at increasingly higher frequencies, which would also be expected. If one follows the $\mathrm{I} / \mathrm{O}$ growth pattern for the beginning and end of the trace, including the nonmonotonicities, it is as if there is a suppressive effect that affects the end of the trace. If one were to shift the solid line to the right to meet the dashed line, the two I/O functions would be similar. One would predict that if the above thinking was true, there should be little difference between the beginning and end of the traces after MEM section, which is the case, as shown in the second column of Figure 7.

With MEMR activation, one would expect to see DPOAE magnitude and phase changes. Observations and measurements in cats indicate that the stapediusmuscle contraction increases the stapes impedance through strain on the annular ligament (Pang and Peak 1986). Other researchers have used increased stapedial impedance in the middle-ear model by Zwislocki (1962) to model how the middle ear changes with stapedius muscle contraction. The middle ear becomes stiffer, which increases middleear impedance below the resonant frequency with a corresponding phase lead (Pascal et al. 1998; Buki et al. 2000). Before MEM section, we see large $\Delta \mathrm{LC}$ and $\triangle \mathrm{PC}$ over a broad range of frequencies as would be predicted from previous work on MEMR in the rat (Murata et al. 1986). We would predict that the MEMR contraction has reduced the effective level of the primaries transmitted to the cochlea and has also reduced the DPOAE traveling through the middle 
ear. From Figure 11, it can be seen that the phase of the DPOAE during contralateral stimulation is relatively constant across L2 level. Below the I/O notch, we observe a phase lead between the onset of the DPOAE trace and the DPOAE phase during contralateral stimulation as would be predicted if the noise was activating the MEMR. Additional phase changes are often observed for the highest primary levels, and it is hypothesized that the primaries are evoking a MEMR, and when the contralateral noise is applied, the MEMR strength increases.

Whereas our data suggest that major contribution to $\triangle \mathrm{LC}$ and $\triangle \mathrm{LI}$ is due to MEMR, we have not performed the control experiment where we left the MEMs intact and cut the MOC fibers. It is extremely difficult in the rat to cut the MOC without inadvertently damaging the stapedius reflex arc as well. Because we have not performed this control, we cannot rule out definitively that the MOC and MEMR act independently from each other. Analysis of the MEMR and MOC systems has led some to conclude that both systems work together to enhance antimasking effects in the cochlea (e.g., Liberman and Guinan 1998). Although remote, it cannot be ruled out that there is a direct signaling between MOC reflexes and MEMRs, such that, by our act of severing the muscles, we have deactivated the MOC reflex. Although we are not aware of any literature describing direct connections between the two reflex arcs, there is evidence indicating that there are direct projections from the cochlear nucleus to the tensor tympani (Ito and Honjo 1988; Itoh et al. 1986). In addition, a body of research by Fritzsch (1999) suggests that auditory efferent fibers derive developmentally from facial nerve motoneurons, and the two tracts lie close to each other in the brainstem.

\section{MECHANISMS UNDERLYING THE DISTORTION PRODUCT OTOACOUSTIC EMISSION AND MEDIAL OLIVOCOCHLEAR PHENOMENA}

The DPOAE I/O functions presented here are typical for rodents; that being, DPOAE level increases with low and moderate primary levels, exhibits a notch, then increases for higher stimulus levels (e.g., Norton and Rubel 1990; Mills 2002; Lukashkin and Russell 2002; Schmeidt and Adams 1981). Notches were generally observed when L2 was 60-70 dB SPL. Sometimes the DPOAE level was low for L2 $=75 \mathrm{~dB}$ SPL, and had we been able to present higher-level stimuli, it is likely that the notch would have been at or above $75 \mathrm{~dB}$ SPL. For high-frequency stimuli, we appeared to only observe the initial, monotonic section of the DPOAE function and did not observe notches. Perhaps if we had been able to present higher stimulus levels, we would have seen notches in these functions as well. Distortion product otoacoustic emission phase behavior was also typical for rodents (Mills 2002; Lukashkin et al. 2002); that being, there were small changes in DPOAE phase for stimulus levels lower than the notch and an approximately $180^{\circ}$ phase change in or close to the notch. A DPOAE generator that can produce this characteristically linked amplitude and phase behavior has been modeled by Lukashkin et al. (2002) as a nonlinear amplifier with a saturating $\mathrm{I} / \mathrm{O}$ characteristic as a result of the mechanoelectrical transduction of the hair cell bundle (Lukashkin and Russell 1999; Lukashkin et al. 2002). They concluded that the MOC effect of either positive or negative $\Delta$ LI could be explained by a single DPOAE generator (Lukashkin and Russell 1999). The current work demonstrates that this behavior is independent of MEMR, as we observed the linked amplitude and phase behavior before and after sectioning the MEMs. Similar to Lukashkin et al. (2002), who changed the input to the hair cells by using furosemide, we changed the input level to the cochlea by changing the middle ear. The result was that the notch was often found at different stimulus levels and had phase characteristics that followed the hypothesized model.

The assumed MOC-mediated DPOAE onset adaptation and contralateral suppression in the rat exhibited small changes in amplitude but minimal changes in phase. Seeing minimal $\Delta \mathrm{PC}$ and $\Delta \mathrm{PI}$, which was mostly limited to the notch region of the $\mathrm{I} / \mathrm{O}$, seems to be most harmonious with the explanation that MOC action is to reduce cochlear amplifier gain. Alternatively, one could argue that the MOC altered both primaries equally, resulting in a reduced DPOAE with minimal phase change. However, given the nontypical $\Delta \mathrm{LC}$ and $\triangle \mathrm{PC}$ results in one animal at one frequency that exhibited no $\Delta \mathrm{LC}$ along with a $\triangle \mathrm{PC}$ phase lead, we cannot rule out that there are two regions generating DPOAEs in the rat, such as that modeled by Shera and Guinan (1999), and that the MOC reflex affected one region more than the other. Although we have not tested for two DPOAE-emitting regions in Sprague-Dawley rats by measuring microstructure, other rodents, such as guinea pigs (Withnell et al. 2003) and kangaroo rats (Long et al. 1999), do have some DPOAE microstructure.

Although the behavior of $\Delta \mathrm{LI}$ and $\Delta \mathrm{LC}$ in the rat is similar with and without intact MEMs, except for a difference in size, $\triangle \mathrm{PI}$ and $\triangle \mathrm{PC}$ are dramatically different under these two conditions. Based on these data, it brings up the question whether observing phase changes along with $\Delta \mathrm{LI}$ and $\triangle \mathrm{LC}$ will help parse out what changes are due to MOC and what may be due to MEMR. Our data lend support to work 
in humans where phase changes are being used to help determine middle-ear muscle contamination of OAE responses (Buki et al. 2000; Guinan et al. 2003).

\section{ACKNOWLEDGMENT}

This work was supported by grant no. 2P01 DC00380 from the National Institute of Deafness and other Communication Disorders.

\section{REFERENCES}

Buki B, Wit HP, Avan P. Olivocochlear efferent vs. middle-ear contributions to the alteration of otoacoustic emissions by contralateral noise. Brain Res. 852:140-150, 2000.

Collet L, Kemp DT, Veuillet E, Duclaux R, Moulin A, Morgon A. Effect of contralateral auditory stimuli on active cochlear micromechanical properties in human subjects. Hear. Res. 43:251261, 1990.

Fritzsch B. Ontogenetic and evolutionary evidence for the motoneuron nature of the vestibular and cochlear efferents. In: Berlin CI (ed) The Efferent Auditory System: Basic Science and Clinical Applications. Singular Publishing Group, Inc., San Diego, pp 31-60, 1999.

Guinan, JJ JR, Backus BC, Lilaonitkul W, Aharonson V. Medial olivocochlear efferent reflex in humans: otoacoustic emission (OAE) measurement issues and the advantages of stimulus frequency OAEs. J. Assoc. Res. Otolaryngol. 4:521-540, 2003.

Iто J, Honjo I. Electrophysiological an dHRP studies of the direct afferent inputs from the cochlear nuclei to the tensor tympani muscle motoneurons in the cat. Acta Otolaryngol. 105:292-296, 1988.

Itoh K, Nomura S, Konishi A, Yasui Y, Sugimoto T, Muzion N. A morphological evidence of direct connections from the cochlear nuclei to tensory tympani motoneurons in the cat: a possible afferent limb of the acoustic middle ear reflex pathways. Brain Res. 375:214-219, 1986.

Jacobson M, Kim S, Romney J, Zhu X, Frisina R. Contralateral suppression of distortion-product otoacoustic emissions declines with age: a comparison of findings in CBA mice with human listeners. Laryngoscope 113:1707-1713, 2003.

Kim DO, Dorn PA, NeEly ST, Gorga MP. Adaptation of distortion product otoacoustic emission in humans. J. Assoc. Res. Otolaryngol. 2:31-40, 2001.

KujaWA SG, Liberman MC. Effects of olivocochlear feedback on distortion product otoacoustic emissions in guinea pig. J. Assoc. Res. Otolarygol. 2:268-278, 2001.

Liberman MC, Puria S, Guinan, JJ Jr. The ipsilaterally evoked olivocochlear reflex causes rapid adaptation of the 2f1-f2 distortion product otoacoustic emission. J. Acoust. Soc. Am. 99:3572-3584, 1996.

Liberman MC, Guinan, JJ Jr. Feedback control of the auditory periphery: anti-masking effects of middle ear muscles vs. olivocochlear efferents. J. Commun. Disord. 6:471-482, 1998.

Long GR, Shaffer LA, Dhar S, Talmadge CL. Cross species comparison of otoacoustic fine structure. In: Wada H, Takasaka T, Ikeda K, Ohyama K, Koike T (eds) Recent Developments in Auditory Mechanics. World Scientific Press, Singapore, pp 367-373, 1999.

Luebke AE, Foster PK, Stagner BB. A multifrequency method for determining cochlear efferent activity. J. Assoc. Res. Otolaryngol. 3:16-25, 2002.

LUKASHKIN AN, RusSELl IJ. Analysis of the f2-f1 and 2f1-f2 distortion components generated by the hair cell mechanoelectrical transducer: dependence on the amplitudes of the primaries and feedback gain. J. Acoust. Soc. Am. 106:2661-2668, 1999.

LuKASHKIN AN, Russell IJ. Modifications of a single saturating nonlinearity account for post-onset changes in $2 \mathrm{f} 1-\mathrm{f} 2$ distortion product otoacoustic emission. J. Acoust. Soc. Am. 112:1561-1568, 2002.

LUKASHKIN AN, LuKASHKINA VA, Russell IJ. One source for distortion product otoacoustic emissions generated by low- and high-level primaries. J. Acoust. Soc. Am. 111:2740-2748, 2002.

Maison SF, Liberman MC. Predicting vulnerability to acoustic injury with a noninvasive assay of olivocochlear reflex strength. J. Neurosci. 20:4701-4707, 2000.

McGee J, Walsh EJ, Neely S, Joseph J, Kim DO. Adaptation of distortion product otoacoustic emissions in gerbils and mice. Abstracts of the Midwinter Meeting of the Association for Research in Otolaryngology, p. 2000.

MiLLS DM. Interpretation of standard distortion product otoacoustic emission measurements in light of the complete parametric response. J. Acoust. Soc. Am. 112:1545-1560, 2002.

Murata K, Ito S, Horikawa J, Minami S. The acoustic middle ear muscle reflex in albino rats. Hear. Res. 23:169-183, 1986.

Norton SJ, Rubel EW. Active and passive ADP components in mammalian and avian ears. In: Dallos P, Geisler CD, Matthews JW, Ruggero JW, Steele CR (eds) Mechanics and Biophysics of Hearing. Springer-Verlag, Berlin Heidelberg New York, pp 219-226, 1990.

Pang XD, Peak WT. How do contractions of the stapedius muscle alter the acoustic properties of the ear? In: Allen JB, Hall JL, Hubbard A, Neely ST, Tubis A (eds) Peripheral Auditory Mechanisms. Springer-Verlag, Berlin Heidelberg New York, pp 36-43, 1986.

Pascal J, Bourgeade A, Lagier M, Legros C. Linear and nonlinear model of the human middle ear. J. Acoust. Soc. Am. 104:1509-1516, 1998.

Puel JL, Rebillard G. Effect of contralateral sound stimulation on the distortion product 2F1-F2: evidence that the medial efferent system is involved. J. Acoust. Soc. Am. 87:1630-1635, 1990.

Rosowski JJ, Peake WT, White JR. Cochlear nonlinearities inferred from two-tone distortion products in the ear canal of the alligator lizard. Hear. Res. 13:141-158, 1984.

Schmeidt RA, Adams JC. Stimulated acoustic emission in the ear canal of the gerbil. Hear. Res. 5:295-305, 1981.

Shera CA, Guinan, JJ JR. Evoked otoacoustic emissions arise by two fundamentally different mechanisms: a taxonomy for mammalian OAEs. J. Acoust. Soc. Am. 105:782-798, 1999.

Siegel JH, Hirohata ET. Sound calibration and distortion product otoacoustic emissions at high frequencies. Hear. Res. 80:146$152,1994$.

Sun XM, Kim DO. Adaptation of 2f1-2f2 distortion product otoacoustic emission in young-adult and old CBA and C57 mice. J. Acoust. Soc. Am. 105:3399-3409, 1999.

Talmadge CL, Long GR, Tubis A, Dhar S. Experimental confirmation of the two-source interference model for the fine structure of distortion product otoacoustic emissions. J. Acoust. Soc. Am. 105:275-292, 1999.

Veuillet E, Collet L, Duclaux R. Effect of contralateral acoustic stimulation on active cochlear micromechanical properties in human subjects: dependence on stimulus variables. J. Neurophysiol. 65:724-735, 1991.

Whitehead ML, Martin GK, Lonsbury-Martin BL. Effects of the crossed acoustic reflex on distortion-product otoacoustic emissions in awake rabbits. Hear. Res. 51:55-72, 1991.

Withnell RH, Shaffer LA, Talmadge CL. Generation of DPOAEs in the guinea pig. Hear. Res. 178:106-117, 2003.

ZwisLocki JJ. Analysis of the middle-ear function. Part I: input impedance. J. Acoust. Soc. Am. 34:1514-1523, 1962. 